\title{
A Multiagent Evolutionary Algorithm for Minimizing Network Coding Resource in Dynamic Environment
}

\author{
Fuhong Song, Huanlai Xing ${ }^{1, \star}$, Zhimin Xia, Yingjie Wang, Shili He, Xingxue \\ Zhou and Yu Zhang
}

${ }^{1}$ School of Information Science and Technology, Southwest Jiaotong University, Chengdu610031, China;

*Corresponding author: hxx@home.swjtu.edu.cn

Keywords: Network coding, dynamic network environment, multiagent evolutionary algorithm.

\begin{abstract}
This paper investigates the network coding resource minimization problem in the context of dynamic network environment. As a combination of multiagent systems and evolutionary algorithm, multiagent evolutionary algorithm (MAEA) is adapted for the above NP-hard problem. Simulation results demonstrate that the proposed MAEA outperforms a number of state-of-the-art evolutionary algorithms with respect to the solution quality.
\end{abstract}

\section{Introduction}

As an advanced communication paradigm, network coding has testified that the theoretical max-flow can always be achieved [1]. However, it is known that performing coding operations may cause heavy computational resource consumption due to the complicated mathematical functions involved. Hence, it is of vital importance to minimize the number of coding operations, which is called the network coding resource minimization (NCRM) problem. Numerous research attention has been dedicated to the above problem, including genetic algorithm (GA) [2], quantum-inspired evolutionary algorithm (QEA) [3] and population based incremental learning (PBIL) [4]. All the work above assumes that the network environment is static so the problem fitness landscape is unchangeable. Nevertheless, the real-world network environment is often dynamic. For instance, network topology might change frequently because of network failures and/or network element leaving/joining. Therefore, it is essential to always keep the number of coding operations minimized in a dynamic network environment. However, to the best of our knowledge, this topic has received little research attention.

Proposed by Liu et al, multiagent evolutionary algorithm integrates multiagent systems and evolutionary algorithm [5]. MAEA has been reported to outperform a number of existing algorithms in terms of global optimization and convergence when applied for large scale deceptive and hierarchical problems. With these properties, agents can respond quickly to environmental changes, hence making MAEAone of idea candidates for dynamic optimization problems.

In this paper, we adapt MAEA for the NCRM problem with dynamic network environment. With specially devised agent behavior, the proposed algorithm can locate the moving optima rapidly in an ever-changing network environment. Simulation results show that the proposed MAEA is better than a number of existing evolutionary algorithms regarding the best solution obtained.

\section{Problem Formulation}

We denote a communication network at time $\tau$ by a directed graph $G(\tau)=\left(V_{\tau}, E_{\tau}\right)$, where $V_{\tau}$ and $E_{\tau}$ is the set of nodes and links, respectively. Suppose each link $e \in E_{\tau}$ has a unit capacity. In a single-source NCM scenario, data information is delivered at date rate $R$ (an integer) from a source node $s \in V_{\tau}$ to a number of receivers $T_{\tau}=\left\{t_{1}, \ldots, t_{d}\right\}, t_{k} \in V_{\tau}, k=1, \ldots, d$. A merging node is a non-receiver intermediate node with multiple incoming links in the original network. Coding operations only occur at merging nodes. Compared to the number of coding nodes, the amount of coding links is more accurate to estimate the computational overhead of coding operations. So, this paper estimates the network coding resource consumption by number of coding links. More details can be found in [2]. 
We call a subgraph in $G(\tau)$ as a network coding based multicast (NCM) subgraph, if there are $R$ link-disjoint paths $\left\{P_{1}\left(s, t_{k}, \tau\right), \ldots, P_{R}\left(s, t_{k}, \tau\right)\right\}$ from $s$ to each receiver $t_{k} \in V_{\tau}$, and denote the subgraph by $G_{N C M}(\tau)$.Let $\Omega(\tau)$ be the number of coding links in $G_{N C M}(\tau)$. In $G_{N C M}(\tau)$, let $\gamma\left(s, t_{k}, \tau\right)$ and $W_{i}\left(s, t_{k}, \tau\right)$ denote the data rate between $s$ and $t_{k}$, and the link set of the $i$-th path from $s$ to $t_{k}, i=1, \ldots$, $R$, respectively.

In this paper, we define the dynamic NCRM problem as to minimize the number of coding links, shown as follows.

Minimize:

$$
\Omega(\tau)
$$

Subject to:

$$
\begin{gathered}
\gamma\left(s, t_{k}, \tau\right) \geq R, \forall t_{k} \in T_{\tau} \\
W_{i}\left(s, t_{k}, \tau\right) \cap W_{j}\left(s, t_{k}, \tau\right)=\varnothing, \forall t_{k} \in T_{\tau}, i \neq j
\end{gathered}
$$

Constraint (2) defines that the achievable data rate from $s$ to each receiver is not smaller than $R$. Constraint (3) indicates that no common link is shared by different paths with the same destination.

\section{The Proposed Algorithm}

Brief introduction of MAEA. This section first simply describes agents in MAEA. Then, the behavior of agents is introduced, includingthe competition and self-learning behaviors [5].

1) Agent: Let $\boldsymbol{a}$ represents an agent which is encoded by a binary vector

$$
\boldsymbol{a}=\left(a_{1}, a_{2}, \ldots, a_{n}\right) \text {. }
$$

The energy of $\boldsymbol{a}$ is set to its associated fitness value, i.e.Energy $(\boldsymbol{a})=f(\boldsymbol{a})$. Agentaaims to increase its energy at its best. Since the dynamic NCRM problem concerned in the paper is a minimization problem, the purpose of $\boldsymbol{a}$ is redefined as to decrease its energy as much as possible.

In MAEA, every agent live in a toroidal lattice like environment, which is referred to as agent lattice and denoted by $\boldsymbol{L}$, where $\boldsymbol{L}=L_{\text {size }} \times L_{\text {size }}$ and $L_{\text {size }}$ is an integer. For an arbitrary agent, it is fixed on a lattice point and it interacts only with its neighbors. Let $\boldsymbol{L}_{i, j}$ represent the agent at point $(i, j), i, j=1, \ldots, L_{\text {size. }}$. Let the neighbors of $\boldsymbol{L}_{i, j}$ denoted by $L_{k, l}$, is determined by a parameter, namely, perception range $\lambda$, as below (5), (6).

$$
\begin{aligned}
& (i-\lambda) \leq k \leq(i+\lambda) \text { and } k=\left\{\begin{array}{l}
k+L_{\text {size }}, k<1 \\
k-L_{\text {size }}, k>L_{\text {size }}
\end{array}\right. \\
& (j-\lambda) \leq l \leq(j+\lambda) \text { and } l=\left\{\begin{array}{l}
l+L_{\text {size }}, l<1 \\
l-L_{\text {size }}, l>L_{\text {size }}
\end{array}\right.
\end{aligned}
$$

2) Competition Behavior: In this paper, the perception range $\lambda$ is fixed to 1 . Thus, each agent owns eight neighbors. For $\boldsymbol{L}_{i, j}$, its energy is first compared with the energies of its neighbors. If $\boldsymbol{L}_{i, j}$ 's energy is the lowest, $\boldsymbol{L}_{i, j}$ survives in the current generation; otherwise, the lattice point of $\boldsymbol{L}_{i, j}$ will be replaced by the child of the neighbor with the lowest energy in the neighborhood of $\boldsymbol{L}_{i, j}$. More details can be found in [5].

3) Self-Learning Behavior: Since we have a minimization problem, we devise this behavior as to reduce the energy of each agent as much as possible. Nevertheless, due to the limitation of resources in the environment, an agent is allowed to get a self-learning opportunity only if it is associated with the smallest energy in the neighborhood. This paper is based on a self-learning strategy with iteratively partial reversing [5], where 10 elements are randomly selected from the original learning table to form a new sub-table. With the new sub-table, the self-learning behavior is performed, which helps decrease the consumed computational overhead.

Overall Procedure. The proposed algorithm is based on binary link state (BLS) representation, which has been widely adopted for NCRM problems [2]. 
For agent evaluation, we first check if agentais feasible. If it results into a feasible NCM subgraph, the fitness value of $\boldsymbol{a}$ is set to the number of coding links in the NCM subgraph; otherwise, itis set to a sufficiently large value.

We hereafter call the proposed algorithm as MAEA for network coding and denote it by NC-MAEA. The overall procedure of NC-MAEA is exhibited in Fig.1.

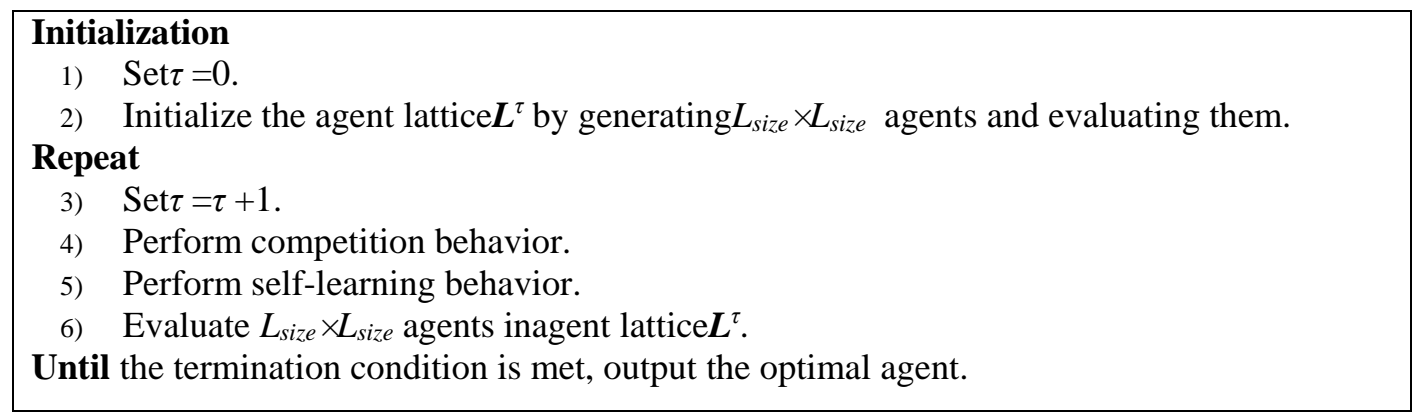

Fig.1 The procedure of NC-MAEA

\section{Performance Evaluation}

In this section, we first introduce all test instances and then compare NC-MAEA with a number of existing evolutionary algorithms in terms of the optimization performance.

Test Instances. We use ten test instances for performance comparison, including six fixed networks and four randomly generated networks. All instances are shown in Table 1.

Table 1 Test Instances and Their Parameters

\begin{tabular}{cccccc}
\hline Network & Nodes & Links & Receivers & Rate & Optima \\
\hline 3-copy & 25 & 36 & 4 & 2 & 0 \\
7-copy & 57 & 84 & 8 & 2 & 0 \\
15-copy & 121 & 180 & 16 & 2 & 0 \\
3-hybrid & 24 & 34 & 4 & 2 & 1 \\
7-hybrid & 55 & 80 & 8 & 2 & 2 \\
15-hybrid & 118 & 174 & 16 & 2 & 3 \\
Rnd-1 & 20 & 37 & 5 & 3 & 0 \\
Rnd-2 & 30 & 69 & 6 & 3 & 0 \\
Rnd-3 & 40 & 78 & 9 & 3 & 0 \\
Rnd-4 & 50 & 101 & 8 & 3 & 0 \\
\hline
\end{tabular}

The dynamic problem generator in [7] is used to generate ten dynamic optimization problems. The network environment changes every 50 generations and for each instance there are 5 changes. Therefore, the predefined number of generations is set to 300 . The size of agent lattice $\left(L_{\text {size }}\right)$ for NC-MAEA is set to 5.So, the population size for each algorithm is set to 25. Algorithms stop ifa predefined number of generations is evaluated.

Overall Performance Comparison. We compare the following six algorithms.

1) GA: genetic algorithm with BLS encoding [2].

2) QEA: quantum-inspired evolutionary algorithm [3].

3) PBIL: traditional population based incremental learning [4].

4) PBILr: population based incremental learning with restart scheme [7].

5) UMDA: univariate marginal distribution algorithm [6].

6) NC-MAEA: the proposed algorithm in the paper.

For performance comparison, each algorithm is run 20 times. For each single run, the best fitness values obtained from each stationary period are averaged, which is called the average best fitness value (ABFV). Thus, after 20 runs, we obtain $20 \mathrm{ABF}$ values for each algorithm. Their mean value (MEAN) and standard deviation (SD) are utilized to show the optimization performance.The 
experimental results areshown in Table 2, with respect to the mean value and standard deviation of ABFV. Obviously, NC-MAEA is better than the other algorithms.

Table 2 The Experimental Results of MEAN and SD (Best Results are Bold)

\begin{tabular}{ccccccc}
\hline Network & GA & QEA & PBIL & PBILr & UMDA & NC-MAEA \\
\hline 3-copy & $0.34(0.24)$ & $0.3(0.22)$ & $0.57(0.42)$ & $0.08(0.11)$ & $1.88(0.77)$ & $\mathbf{0}(0)$ \\
7-copy & $2.94(0.99)$ & $5.71(1.17)$ & $2.23(0.90)$ & $1.09(0.43)$ & $7.13(0.91)$ & $\mathbf{0}(0)$ \\
15-copy & $14.93(1.39)$ & $27.74(3.36)$ & $9.81(1.15)$ & $14.15(0.82)$ & $22.92(1.40)$ & $\mathbf{2 . 0 8}(0.80)$ \\
3-hybrid & $1.36(1.20)$ & $1.24(0.30)$ & $1.45(0.41)$ & $1.01(0.04)$ & $2.49(0.57)$ & $\mathbf{1}(0)$ \\
7-hybrid & $5.51(1.00)$ & $6.72(1.15)$ & $3.76(0.75)$ & $2.72(0.33)$ & $8.30(0.97)$ & $\mathbf{2}(0)$ \\
15-hybrid & $16.67(0.92)$ & $31.02(2.02)$ & $12.17(1.66)$ & $15.94(0.99)$ & $26.73(2.64)$ & $\mathbf{4 . 1 7}(0.45)$ \\
Rnd-1 & $0.83(0.18)$ & $0.36(0.21)$ & $0.65(0.28)$ & $0.38(0.21)$ & $1.08(0.27)$ & $\mathbf{0 . 0 5}(0.09)$ \\
Rnd-2 & $0.04(0.07)$ & $0.03(0.06)$ & $0.05(0.09)$ & $\mathbf{0}(0)$ & $0.29(0.19)$ & $\mathbf{0}(0)$ \\
Rnd-3 & $1.66(0.29)$ & $1.47(0.41)$ & $1.18(0.42)$ & $0.81(0.25)$ & $2.13(0.42)$ & $\mathbf{0 . 4 4}(0.24)$ \\
Rnd-4 & $0.58(0.23)$ & $0.59(0.23)$ & $0.38(0.30)$ & $0.21(0.16)$ & $1.16(0.43)$ & $\mathbf{0 . 0 4}(0.09)$ \\
\hline
\end{tabular}

\section{Conclusion}

This paper studies the network coding resources minimization problem in a dynamic network environment and provides a multiagent evolutionary algorithm to address it. The performance evaluation clearly demonstrates the superiority of the proposed algorithm over a number of existing evolutionary algorithms with respect to the solution quality.

\section{Acknowledgment}

This work was supported in part by National Natural Science Foundation of China (No.61401374), the Project-sponsored by SRF for ROCS, SEM, P. R. China.

\section{References}

[1] S. Y. R. Li, R. W. Yeung, N. Cai. Linear network coding. IEEE Transactions on Information Theory, 2003, vol. 49, no. 2, pp. 371-381.

[2] M. Kim, M. Médard, V. Aggarwal, et al. Evolutionary approaches to minimizing network coding resources. Proceedings of 26th IEEE International Conference on Computer Communications (INFOCOM 2007). 2007, pp. 1991-1999.

[3] H. Xing, Y. Ji, Li. Bai and Y. Sun. An improved quantum-inspired evolutionary algorithm for coding resource optimization based network coding multicast scheme. AEU - International Journal of Electronics and Communications, 2010, vol. 64, pp. 1105-1113.

[4] H. Xing and R. Qu. A population based incremental learning for network coding resources minimization. IEEE Comm. Lett, 2011. Vol. 15, 698-700.

[5] J. Liu, W. Zhong and L. Jiao. A multiagent evolutionary algorithm for combinatorial optimization problems. IEEE Transactions on systems, man, and cybernetics.2010, Vol. 40, no. 1, pp, 229-240.

[6] L. V. Lozada-Chang and R.Santana. Univatiate marginal distribution algorithm dynamics for a class of parametric functions with unitation constrains. Info.Sci. 2011, vol. 181, pp. 2340-2355.

[7] S. Yang and X. Yao. Experiment study on population-based incremental learning algorithms for dynamic optimization problems. Soft computing, 2005, vol. 9, no. 11, pp. 815-834. 